\title{
Interaction of FUN14 domain containing 1, a mitochondrial outer membrane protein, with kinesin light chain 1 via the tetratricopeptide repeat domain
}

\author{
WON HEE JANG ${ }^{1}$, YOUNG JOO JEONG ${ }^{1}$, SUN HEE CHOI ${ }^{1}$, SANG-HWA URM ${ }^{2}$ and DAE-HYUN SEOG ${ }^{1}$ \\ Departments of ${ }^{1}$ Biochemistry and ${ }^{2}$ Preventive Medicine, \\ Inje University College of Medicine, Busan 614-735, Republic of Korea
}

Received October 20, 2016; Accepted November 9, 2016

DOI: $10.3892 /$ br.2016.818

\begin{abstract}
Kinesin 1 is a member of the kinesin superfamily proteins (KIFs) of microtubule-dependent molecular motor proteins that transport organelles and protein complexes in cells. Kinesin 1 consists of a homo- or hetero-dimer of kinesin heavy chains (KHCs), often, although not always, associated with two kinesin light chains (KLCs). KLCs are non-motor proteins that associate with many different binding proteins and cargoes, but their binding partners have not yet been fully identified. In the present study, a yeast two-hybrid system was used to identify proteins that interact with the tetratricopeptide repeat (TPR) domain of KLC1. The results of the current study revealed an interaction between the TPR domain of KLC1 and FUN14 domain-containing protein 1 (FUNDC1), which is a mitochondrial outer membrane protein mediating hypoxia-induced mitophagy. FUNDC1 bound to the six TPR motif-containing regions of $\mathrm{KLC} 1$ and did not interact with KIF5B (a motor subunit of kinesin 1) and KIF3A (a motor subunit of kinesin 2) in the yeast two-hybrid assay. The cytoplasmic amino N-terminal domain of FUNDC1 is essential for interaction with KLC1. When co-expressed in HEK-293T cells, FUNDC1 co-localized with KLC1 and co-immunoprecipitated with KLC1, but not KIF5B. Collectively, these results indicate that KLC1 may potentially compete with LC3, a key component for autophagosome formation, to interact with FUNDC1.
\end{abstract}

\section{Introduction}

Kinesin-mediated cargo transport is essential for many cellular functions and the morphogenesis of cells. Kinesin superfamily proteins (KIFs) are microtubule-dependent motor

Correspondence to: Professor Dae-Hyun Seog, Department of Biochemistry, Inje University College of Medicine, 75 Bokji-ro, Busanjin-gu, Busan 614-735, Republic of Korea

E-mail: daehyun@inje.ac.kr

Key words: FUN14 domain containing 1, kinesin 1, kinesin light chain 1 , microtubule motors, tetratricopeptide repeat domain proteins involved in the transport of various cargoes, including membrane vesicles, organelles, proteins complexes, and mRNAs $(1,2)$. Kinesin 1 is the first identified member of KIFs responsible for anterograde axonal transport (3). Kinesin 1 is a tetrameric protein composed of two KIF5 motors, also known as kinesin heavy chains (KHCs) and two kinesin light chains (KLCs) (2-4). The KIF5s contain the motor domain at the amino N-terminal region, a coiled-coil dimerization domain, and a carboxyl C-terminal tail region that regulates the motor activity. KLCs contain the $\mathrm{N}$-terminal $\alpha$-helix domain that binds to KIF5s, the central tetratricopeptide repeat (TPR) domains, and the $\mathrm{C}$-terminal region that negatively regulates the motor domain ATPase activity of KIF5s (3-5). Three isoforms of KIF5s exist in mammals; KIF5A and KIF5C are expressed in neurons, whereas KIF5B is expressed ubiquitously (6). Three isoforms of KLC have been identified in mammals as follows: The neuronal tissue-specific KLC1, the ubiquitous KLC2, and the testis-specific KLC3 (4,7). Certain kinesin 1 cargoes, such as glutamate receptor-interacting protein 1 and $\gamma$-aminobutyric acid receptor-associated protein interact directly with the tail cargo binding-domain of KIF5s, while others, including S100 proteins and Alcadein $\alpha$ bind to the TPR domains of KLCs (8-11).

The TPR domain consisting of multiple tandem repeats is a well-known module facilitating protein-protein interaction that organizes complexes involved in a number of biological processes, including the adaptor function that the binding kinesin 1 to other cargos $(12,13)$. Each TPR motif consists of 34 amino acids, which form a helix-turn-helix structure (12). The TPR domains of KLCs are highly conserved across species and are known to be involved in cargo interaction $(5,12-14)$. The first protein identified to bind to the TPR domain of KLC1 was the c-Jun NH2-terminal kinase (JNK)-interacting protein (JIP, also termed JSAP) group $(15,16)$. The three JIP isoforms in mammals are scaffolds for the mitogen-activated protein kinase cascade that activates JNK $(17,18)$.

Although many cargoes, including organelles moved by kinesin 1 have been identified, it remains unclear as to how kinesin 1 binds to different cargoes and regulates organelle transport. In certain cases, cargoes bind to adaptor/scaffolding proteins that mediate the attachment of kinesin 1 to the cargo (2). To improve the understanding of kinesin 1-dependent transport 
events, it was necessary to further identify the interacting partners of kinesin 1 . In the present study, proteins that interact specifically with the TPR domain of KLC1 were screened for and FUN14 domain-containing protein 1 (FUNDC1), which is a mitochondrial outer membrane protein with three transmembrane domains (19) was identified. FUNDC1 functions as a receptor of selective mitophagy in response to hypoxia via interaction with LC3 (19). The interaction between KLC1 and FUNDC1 indicates that KLC1 may serve as a potential competitor to LC3 for interaction with FUNDC1.

\section{Materials and methods}

Plasmid constructs. The mouse KLC1 cDNA fragment corresponding to the TPR domain (amino acids 80-541) was amplified by polymerase chain reaction (PCR) from the Marathon-Ready ${ }^{\mathrm{TM}}$ cDNA library (Clontech Laboratories, Inc., Palo Alto, CA, USA) using the appropriate primers (forward primer: 5'-AGCGAGGCGCAGGTGATGATG GCG-3', reverse primer: 5'-AGTGCCATCCCCATTCCACTC TAC-3'). The PCR reaction was performed in a total volume of $25 \mu \mathrm{l}$ containing $\sim 50 \mathrm{ng}$ cDNA library, $200 \mathrm{mM}$ deoxynucleotide triphosphates (dNTPs), $1 \mathrm{X}$ reaction buffer (MBI Fermentas, St. Leon-Rot, Germany), $1.5 \mathrm{mM} \mathrm{MgCl}_{2}$, and 2-unit Taq polymerase (MBI Fermentas). The temperature profile for the 30 cycles amplification reaction using a PerkinElmer's PCR machine (PerkinElmer Applied Biosystems, Warrington, UK) was as follows: Initial denaturation at $94^{\circ} \mathrm{C}$ for $4 \mathrm{~min}$, denaturation at $94^{\circ} \mathrm{C}$ for $30 \mathrm{sec}$, annealing at $52^{\circ} \mathrm{C}$ for $50 \mathrm{sec}$ and extension at $72^{\circ} \mathrm{C}$ for $2 \mathrm{~min}$, with a final extension at $72^{\circ} \mathrm{C}$ for $5 \mathrm{~min}$. The amplified fragment was cloned into pLexA (Clontech Laboratories, Inc.). The resulting recombinant plasmid, pLexA-6xTPR-KLC1, served as bait plasmid. The full-length cDNA of mouse LC3B (GeneBank accession number: NM_026160) was amplified by PCR from the Marathon-Ready ${ }^{\mathrm{TM}}$ cDNA library (Clontech Laboratories, Inc.) using the appropriate primers (forward primer: 5'-ATGCCGTCCGAGAAGACCTTCAAGCAG-3', reverse primer: 5'-TTACACAGCCATTGCTGTCCCGAA TGT-3') and cloned into pLexA and pB42AD (Clontech Laboratories, Inc.). The PCR reaction was performed according to the above-mentioned method. The C-terminal region of KIF3A (provided by Professor Kozo Kaibuchi; Nagoya University Graduate School of Medicine, Nagoya, Japan) was amplified by PCR using the appropriate primers (forward primer: 5'-CGCCAGTTTCAGAAAGAAATCGAA-3', reverse primer: 5'-TTACTGAAGTAAAGAATCAATTAC-3') and cloned into $\mathrm{pLexA}$ and $\mathrm{pB} 42 \mathrm{AD}$. The PCR reaction was performed according to the above-mentioned method.

Screening of KLC1-binding proteins by yeast two-hybrid assay. The Matchmaker LexA Two-Hybrid system (Clontech Laboratories, Inc.) was used for screening according to the manufacturer's instructions. Briefly, pLexA-6xTPR-KLC1 was transformed into the yeast strain, EGY48 carrying the p8op-lacZ gene. The transformed EGY48 yeast cells containing pLexA-6xTPR-KLC1 were transformed with the mouse brain cDNA library (9) and grown on synthetic dextrose (SD) plates supplemented with glucose, but with no histidine, tryptophan, or uracil (SD/-His/-Trp/-Ura) (Clontech
Laboratories, Inc.). The selection of positive clones was performed on an SD/-His/-Trp/-Ura/-Leu plate containing galactose, raffinose, BU salts (Clontech Laboratories, Inc.) and $0.5 \mathrm{ml}$ of X-Gal solution $(20 \mathrm{mg} / \mathrm{ml})$. Plasmids from the positive clones were analyzed by restriction digestion with EcoRI and XhoI. Unique inserts were sequenced and DNA sequence analysis was performed using the BLAST algorithm (National Center for Biotechnology Information; https://blast.ncbi.nlm. nih.gov/Blast.cgi). Sequence-verified clones were analyzed again for interaction with bait in yeast by retransformation.

$\beta$-Galactosidase activity in liquid cultures of yeast. The $\beta$-galactosidase activity of yeast was assayed as described previously (10). Briefly, mid-log phase yeast cells were collected and permeabilized with $0.1 \%$ sodium dodecyl sulfate (SDS) and chloroform. An excess quantity of o-nitrophenyl- $\beta$-D-galactoside was added to the yeast lysate, the mixture was incubated at $30^{\circ} \mathrm{C}$ and the reaction was terminated by increasing the $\mathrm{pH}$ to 11 by the addition of $1 \mathrm{M}$ $\mathrm{Na}_{2} \mathrm{CO}_{3}$. Formation of the reaction product, o-nitrophenol, was determined by measuring absorbance at a wavelength of $420 \mathrm{~nm}$ on a spectrophotometer, and normalizing for the reaction time and cell density. The units of enzyme activity were calculated with the following equation: Units $=1,000 \times$ [optical density; $\left.\left.\mathrm{OD}_{420}-1.75 \times \mathrm{OD}_{550}\right)\right] /[$ reaction time $(\mathrm{min}) \times$ culture volume $\left.(\mathrm{ml}) \times \mathrm{OD}_{600}\right]$. All experiments were independently performed a minimum of three times.

Glutathione S-transferase (GST) pull-down assays. cDNA encoding the full-length FUNDC1 was cloned into pET41a. The recombinant GST-FUNDC1 fusion protein was expressed in E. coli strain BL21 GOLD (Stratagene, La Jolla, CA, USA) following induction with $0.5 \mathrm{mM}$ isopropyl thio- $\beta$-D-galactopyranoside (IPTG) for $3 \mathrm{~h}$. The fusion proteins were purified by attachment to glutathione-agarose beads (Sigma-Aldrich, St. Louis, MO, USA) according to the manufacturer's protocol. Purified His-tagged KLC1 protein was incubated overnight at $4^{\circ} \mathrm{C}$ with the glutathione beads coupled with GST alone or GST-FUNDC1 protein. The beads were pelleted by centrifugation, washed three times with the extraction buffer [1\% Triton X-100 in phosphate-buffered saline (PBS) containing $10 \mu \mathrm{g} / \mathrm{ml}$ each of aprotinin, leupeptin, and pepstatin and $1 \mu \mathrm{M}$ phenylmethanesulfonyl fluoride], and once with PBS. The bound proteins were eluted from the glutathione beads with $100 \mu 1$ 1X Laemmli loading buffer. The pulled-down proteins were analyzed by immunoblotting with anti-KLC1 antibody (1:800, cat. no. ab187179; Abcam, Cambridge, MA, USA).

Cell culture and transfection. Human embryonic kidney (HEK)-293T [American Type Culture Collection (ATCC) CRL-3216] cells were cultured in Dulbecco's modified Eagle's medium supplemented with $10 \%$ fetal bovine serum, L-glutamine, $100 \mathrm{U} / \mathrm{ml}$ penicillin, and $0.1 \mathrm{mg} / \mathrm{ml}$ streptomycin at $37^{\circ} \mathrm{C}$ in a humidified $5 \% \mathrm{CO}_{2}$ incubator. Transient transfections were performed using the $\mathrm{CaPO}_{4}$ precipitation method (20).

Immunocytochemistry. HEK-293T cells grown on poly-D-lysine-coated coverslips were transfected with enhanced green fluorescent protein (EGFP)-FUNDC1 and KLC1 constructs. Twenty-four hours after transfection, cells 
A

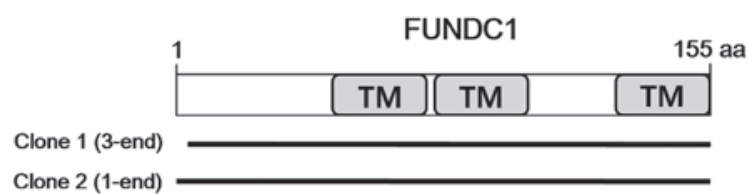

B

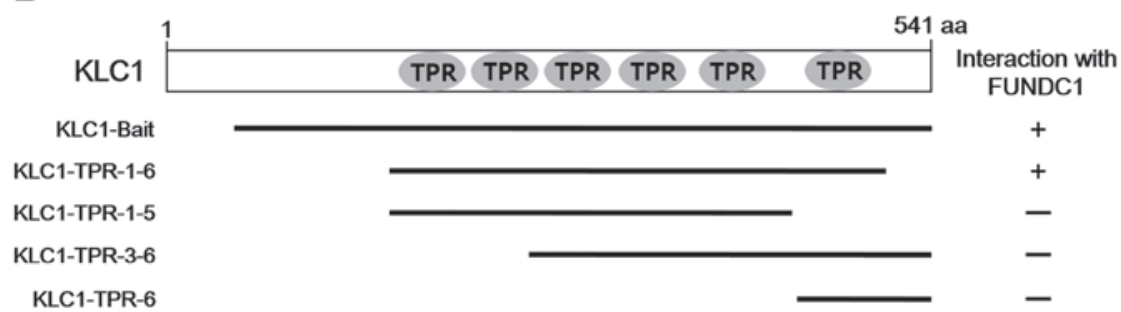

C

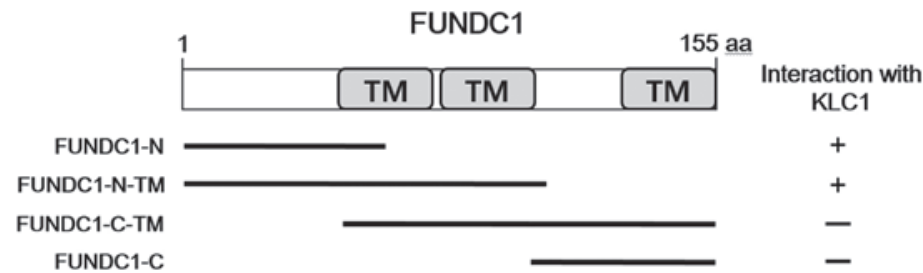

Figure 1. Identification of the proteins interacting with KLC1 by yeast two-hybrid screening. (A) Schematic diagram of FUNDC1. The gray boxes indicate the TM domains. Two positive clones isolated from the yeast two-hybrid screening possess the cDNA for FUNDC1. (B) FUNDC1 binding region in KLC1. KLC1 has six TPR motifs, indicated in gray. The truncated forms of KLC1 were assessed in the yeast two-hybrid assay for interaction with FUNDC1. (C) KLC1 binding region in FUNDC1. Different truncations of FUNDC1 were tested in the yeast two-hybrid assay for interaction with KLC1. +, interaction; -, no interaction; KLC, kinesin light chain; FUNDC1, FUN14 domain-containing protein 1; TM, transmembrane; TPR, tetratricopeptide repeat; aa, amino acids.

were washed with PBS, fixed with $4 \%$ paraformaldehyde in PBS for $5 \mathrm{~min}$, and permeabilized with $0.2 \%$ Triton $\mathrm{X}-100$ (Sigma-Aldrich) in PBS for $10 \mathrm{~min}$. After blocking with $5 \%$ normal goat serum in PBS for $30 \mathrm{~min}$, cells were incubated overnight at $4^{\circ} \mathrm{C}$ with anti-KLC1 antibody (1:500, cat. no. ab187179; Abcam) in PBS containing $1 \%$ bovine serum albumin (BSA) and 0.05\% Tween-20 (Sigma-Aldrich). After washing three times with PBS, cells were incubated for 40 min with Dylight 594-conjugated goat anti-rabbit IgG antibody (1:800, cat. no. 111-516-046; Jackson ImmunoResearch Laboratories, Inc., West Grove, PA, USA). After washing three times with PBS, the cells were mounted with Fluoromount (DAKO, Santa Clara, CA, USA). Fluorescence images were acquired on a Zeiss LSM510 META confocal laser-scanning microscope (Carl Zeiss Inc., Zena, Germany).

Co-immunoprecipitation and immunoblot analysis. Twenty-four hours after transfection with the myc-KLC1 and FLAG-FUNDC1 constructs, the HEK-293T cells were rinsed with ice-cold PBS twice and lysed with ice-cold lysis buffer [PBS containing $0.5 \%$ NP-40 and $1 \mathrm{X}$ protease inhibitor cocktail set V (EMD Millipore, Billerica, MA, USA)] by gentle rotation for $30 \mathrm{~min}$. Lysates were centrifuged at 16,000 x g for $10 \mathrm{~min}$ at $4^{\circ} \mathrm{C}$. The supernatant was incubated with anti-FLAG M2 agarose beads (Sigma-Aldrich) for $2 \mathrm{~h}$ at $4^{\circ} \mathrm{C}$ with constant shaking. The beads were collected by centrifugation at 2,000 x g for $30 \mathrm{sec}$ and washed five times with ice-cold PBS containing $0.5 \%$ NP-40. The washed beads were resuspended with 2X Laemmli loading buffer and the proteins were eluted and denatured by boiling for $2 \mathrm{~min}$. The proteins were processed for $10 \%$ SDS-PAGE and immunoblot analysis with antibodies against KLC1 (Abcam), KIF5B (6), LC3B (1:1,000, cat. no. ab48394; Abcam) and FLAG (1:2,000, cat. no. F7425; Sigma-Aldrich).

\section{Results}

Kinesin 1 is significant in fast axonal transport and is involved in the intracellular trafficking of various cargoes $(1,2)$. Certain cargoes, such as JIPs interact with KLC subunits, but not KHC motor subunits of kinesin $1(15,21)$. To identify proteins that interact with the TPR domain of KLC1, a bait construct encoding a fusion protein containing the TPR domain of mouse KLC1

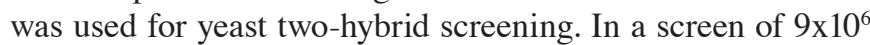
independent transformants, two positive clones were obtained. The two clones overlapped at the open reading frame (ORF) of FUNDC1 (Fig. 1A). To identify the region of KLC1 required for interaction with FUNDC1, various fragments of KLC1 were constructed and tested for interaction with FUNDC1 using a yeast two-hybrid system (Fig. 1B). The result indicates that the region containing all six TPR repeats of KLC1 is required for binding. FUNDC1 is a mitochondrial outer membrane protein with three transmembrane domains (19). To determine the binding domain of FUNDC1 that is required for the interaction with KLC1, various fragments of FUNDC1 were constructed. As shown in Fig. 1C, the N-terminal cytoplasmic region of FUNDC1 interacted with KLC1. Subsequently, whether KLC2, KIF5B, and KIF3A, a motor subunit of kinesin 2, interact with FUNDC1 was investigated. As shown in Fig. 2A, KIF5B and KIF3A did not interact with FUNDC1, but KLC2 bound to 
A

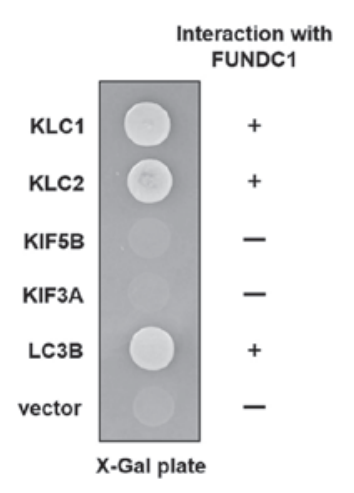

B

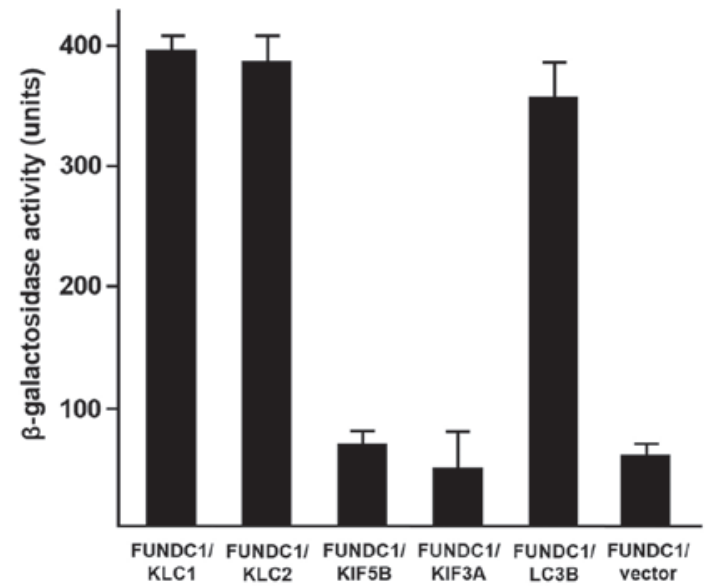

C

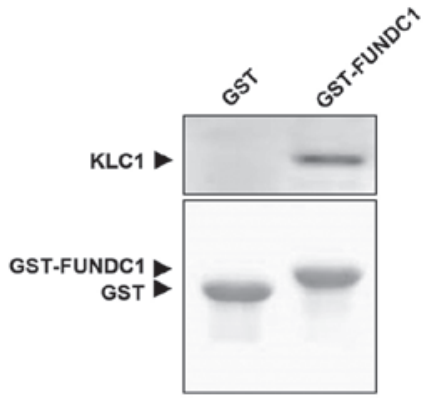

Figure 2. Interaction of KLC1 or KIFs with FUNDC1. (A) The tail regions of each KIF and the full length LC3B and KLCs were tested for the interaction with FUNDC1 in the yeast two-hybrid system. FUNDC1 specifically interacted with KLCs, but not with KIFs. LC3B served as a positive control for interaction. (B) The strength of interactions between FUNDC1 and KIFs, LC3B, or KLCs was examined quantitatively using $\beta$-galactosidase activity in the yeast two-hybrid reporter assay. Values are presented as the mean \pm standard deviation. (C) Direct binding of FUNDC1 to KLC1 in a GST pull-down assay using purified GST-fused FUNDC1 and His-tagged KLC1. +, interaction; -, no interaction; KLC, kinesin light chain; KIF, kinesin superfamily proteins; FUNDC1, FUN14 domain-containing protein 1; GST, glutathione S-transferase.

A

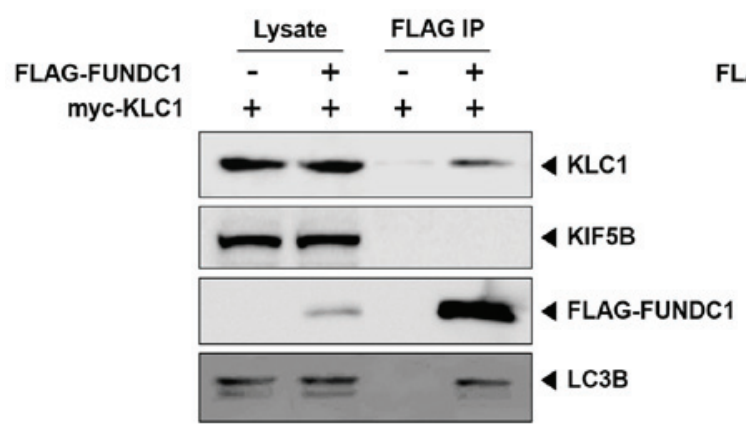

B

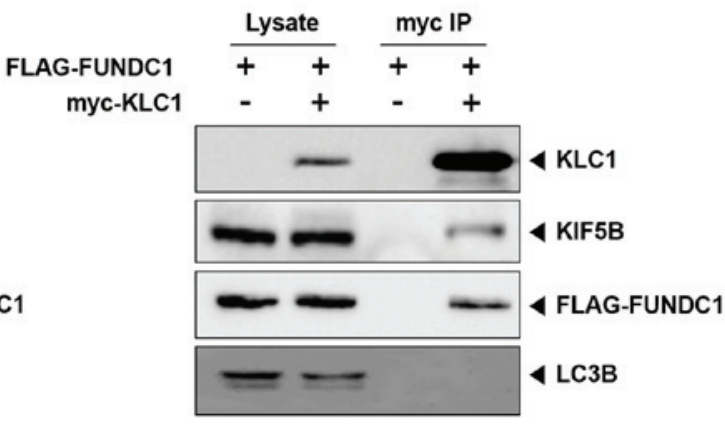

C
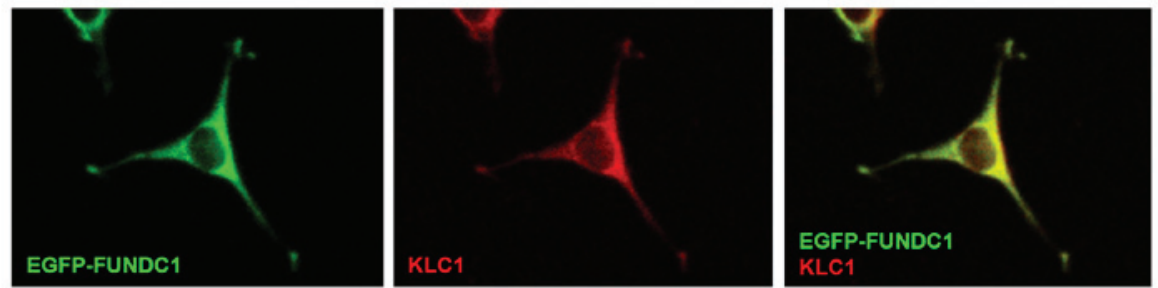

Figure 3. Co-immunoprecipitation and co-localization of KLC1 and FUNDC1 in mammalian cells. (A and B) HEK-293T cells were transiently transfected with FLAG-FUNDC1 and myc-KLC1 plasmids as indicated. Cell lysates were immunoprecipitated with (A) monoclonal anti-FLAG antibody or (B) anti-myc antibody. Precipitates were immunoblotted with anti-KLC1, KIF5B, LC3 and FLAG antibodies. FUNDC1 specifically co-precipitated KLC1 and LC3B, although not KIF5B. (C) HEK-293T cells were transiently transfected with EGFP-FUNDC1 and KLC1 plasmids. Twenty-four hours after transfection, cells were subjected to immunofluorescence with anti-KLC1 antibody. KLC1 and FUNDC1 were observed in the same subcellular region in cells. KLC, kinesin light chain; FUNDC1, FUN14 domain-containing protein 1; KIF, kinesin superfamily proteins; EGFP, enhanced green fluorescent protein; IP, immunoprecipitation.

FUNDC1. LC3B, known to interact with FUNDC1 (19), served as a positive control. In addition, a quantitative $\beta$-galactosidase assay demonstrated that FUNDC1 bound to KLC1 and KLC2 (Fig. 2B). To demonstrate the direct interaction between KLC1 and FUNDC1, GST-FUNDC1 and His-KLC1 proteins were prepared and assessed for binding in a GST pull-down assay. KLC1 interacted with GST-FUNDC1, but not with GST alone (Fig. 2C). This result indicates that KLC1 directly interacts with FUNDC1.
To further confirm the FUNDC1 and KLC1 interaction in mammalian cells, co-immunoprecipitation from HEK-293T cells that were transfected with FLAG-FUNDC1 and myc-KLC1 was performed. Anti-FLAG antibody precipitated KLC1 and endogenous LC3; however, KIF5B did not (Fig. 3A). Conversely, anti-myc antibody precipitated KIF5B and FUNDC1, but not LC3 (Fig. 3B). These results indicate that FUNDC1 interacts with free KLC1, but not with KLC1 bound to KIF5. In order to address whether KLC1 and FUNDC1 co-localize in cells, 
KLC1 was co-expressed with EGFP-FUNDC1 in HEK-293T cells. KLC1 and FUNDC1 were identified to co-localize at the same region in cells (Fig. 3C). Taken together, these results indicate that FUNDC1 is a novel binding partner of $\mathrm{KLCl}$.

\section{Discussion}

Mitochondrial autophagy, mitophagy, is involved in the removal of dysfunctional mitochondria, and controls mitochondrial quality and quantity $(22,23)$. FUNDC1 is a receptor of mitophagy in response to hypoxia (19). LC3 functions as a binding partner for autophagy receptors $(22,23)$. The $\mathrm{N}$-terminal region of FUNDC1 is exposed to the cytosol and contains a typical LC3-interacting region (LIR) motif (19). The conserved LIR motif is essential for the interaction between FUNDC1 and LC3. Deletion or mutations of LIR motif abolish the FUNDC1 and LC3 interaction, and block the induction of mitophagy (19). In the current study, KLC1 interacted with the LIR motif-containing region of FUNDC1. Although the possibility that $\mathrm{KLC} 1$ interacts with another $\mathrm{N}$-terminal region than the LIR motif of FUNDC1 cannot be excluded, this result indicates that the KLC1 may interfere with LC3 binding to FUNDC1. As shown in Fig. 3B, LC3B did not co-precipitate with KLC1-bound FUNDC1, indicating a possible competition between $\mathrm{KLC} 1$ and LC3 for binding to FUNDC1.

The role of adaptor or scaffolding proteins effectively controls the cargo recognition of motors (2). Cargo-associated KIF5s may not always be associated with KLCs. The axonal transport of mitochondria is directly mediated by KLC-independent interaction between the mitochondrial rho (Miro)-Milton complex and KIF5 (24,25). Milton acts as an adaptor protein that links KIF5 motor to Miro, a mitochondrial outer membrane protein $(24,25)$. KLC is not required for the transport of mitochondria and is absent from Milton-KIF5 complex (24). Instead, KLC inhibits the KIF5 and Milton interaction (24). In the present study, FUNDC1 was found to associate with free KLC1, but not KIF5-bound KLC1 (i.e., the $\mathrm{KLC} 1$ as a subunit of kinesin 1). Thus, it is hypothesized that, under normoxia, the KLC1 and FUNDC1 interaction may prevent mitophagy of healthy mitochondria, by inhibiting the FUNDC1 interaction with LC3, and lead to dissociation of KIF5 from KLC1, allowing free KIF5 to associate with the Miro-Milton complex and mediate transport of mitochondria to their proper location. In response to hypoxia, FUNDC1 may be dissociated from KLC1, and subsequently interact with LC3 and trigger mitophagy of damaged mitochondria.

\section{Acknowledgements}

The present study was supported by the Basic Science Research Program through the National Research Foundation of Korea by the Ministry of Education, Science and Technology (grant no. 2015R1D1A1A01056820).

\section{References}

1. Hirokawa N, Niwa S and Tanaka Y: Molecular motors in neurons: Transport mechanisms and roles in brain function, development, and disease. Neuron 68: 610-638, 2010.

2. Fu MM and Holzbaur EL: Integrated regulation of motor-driven organelle transport by scaffolding proteins. Trends Cell Biol 24: 564-574, 2014.
3. Hirokawa N: Kinesin and dynein superfamily proteins and the mechanism of organelle transport. Science 279: 519-526, 1998.

4. Vale RD and Fletterick RJ: The design plan of kinesin motors. Annu Rev Cell Dev Biol 13: 745-777, 1997.

5. Yip YY, Pernigo S, Sanger A, Xu M, Parsons M, Steiner RA and Dodding MP: The light chains of kinesin-1 are autoinhibited. Proc Natl Acad Sci USA 113: 2418-2423, 2016.

6. Kanai Y, Okada Y, Tanaka Y, Harada A, Terada S and Hirokawa N: KIF5C, a novel neuronal kinesin enriched in motor neurons. J Neurosci 20: 6374-6384, 2000.

7. Rahman A, Friedman DS and Goldstein LS: Two kinesin light chain genes in mice. Identification and characterization of the encoded proteins. J Biol Chem 273: 15395-15403, 1998.

8. Setou M, Seog DH, Tanaka Y, Kanai Y, Takei Y, Kawagishi M and Hirokawa N: Glutamate-receptor-interacting protein GRIP1 directly steers kinesin to dendrites. Nature 417: 83-87, 2002.

9. Nakajima K, Yin X, Takei Y, Seog DH, Homma N and Hirokawa N: Molecular motor KIF5A is essential for GABA(A) receptor transport, and KIF5A deletion causes epilepsy. Neuron 76: 945-961, 2012.

10. Shimamoto S, Takata M, Tokuda M, Oohira F, Tokumitsu H and Kobayashi R: Interactions of S100A2 and S100A6 with the tetratricopeptide repeat proteins, Hsp90/Hsp70-organizing protein and kinesin light chain. J Biol Chem 283: 28246-28258, 2008.

11. Araki Y, Kawano T, Taru H, Saito Y, Wada S, Miyamoto K, Kobayashi H, Ishikawa HO, Ohsugi Y, Yamamoto T, et al: The novel cargo Alcadein induces vesicle association of kinesin-1 motor components and activates axonal transport. EMBO J 26: 1475-1486, 2007.

12. Zhu H, Lee HY, Tong Y, Hong BS, Kim KP, Shen Y, Lim KJ, Mackenzie F, Tempel W and Park HW: Crystal structures of the tetratricopeptide repeat domains of kinesin light chains: Insight into cargo recognition mechanisms. PLoS One 7: e33943, 2012.

13. Kawano T, Araseki M, Araki Y, Kinjo M, Yamamoto T and Suzuki T: A small peptide sequence is sufficient for initiating kinesin-1 activation through part of TPR region of KLC1. Traffic 13: 834-848, 2012.

14. Blatch GL and Lässle M: The tetratricopeptide repeat: A structural motif mediating protein-protein interactions. BioEssays 21: 932-939, 1999.

15. Verhey KJ, Meyer D, Deehan R, Blenis J, Schnapp BJ, Rapoport TA and Margolis B: Cargo of kinesin identified as JIP scaffolding proteins and associated signaling molecules. J Cell Biol 152: 959-970, 2001.

16. Sato T, Ishikawa M, Mochizuki M, Ohta M, Ohkura M, Nakai J, Takamatsu N and Yoshioka K: JSAP1/JIP3 and JLP regulate kinesin-1-dependent axonal transport to prevent neuronal degeneration. Cell Death Differ 22: 1260-1274, 2015.

17. Jaeschke A, Czech MP and Davis RJ: An essential role of the JIP1 scaffold protein for JNK activation in adipose tissue. Genes Dev 18: 1976-1980, 2004

18. Ito M, Yoshioka K, Akechi M, Yamashita S, Takamatsu N, Sugiyama K, Hibi M, Nakabeppu Y, Shiba T and Yamamoto KI: JSAP1, a novel jun N-terminal protein kinase (JNK)-binding protein that functions as a Scaffold factor in the JNK signaling pathway. Mol Cell Biol 19: 7539-7548, 1999.

19. Liu L, Feng D, Chen G, Chen M, Zheng Q, Song P, Ma Q, Zhu C, Wang R, Qi W, et al: Mitochondrial outer-membrane protein FUNDC1 mediates hypoxia-induced mitophagy in mammalian cells. Nat Cell Biol 14: 177-185, 2012.

20. Wigler M, Silverstein S, Lee LS, Pellicer A, Cheng Y and Axel R: Transfer of purified herpes virus thymidine kinase gene to cultured mouse cells. Cell 11: 223-232, 1977.

21. Cyrus BF and Muller WA: A unique role for endothelial cell kinesin light chain 1 , variant 1 in leukocyte transendothelial migration. Am J Pathol 186: 1375-1386, 2016.

22. Wei H, Liu L and Chen Q: Selective removal of mitochondria via mitophagy: distinct pathways for different mitochondrial stresses. Biochim Biophys Acta 1853: 2784-2790, 2015.

23. Murrow L and Debnath J: Autophagy as a stress-response and quality-control mechanism: Implications for cell injury and human disease. Annu Rev Pathol 8: 105-137, 2013.

24. Glater EE, Megeath LJ, Stowers RS and Schwarz TL: Axonal transport of mitochondria requires milton to recruit kinesin heavy chain and is light chain independent. J Cell Biol 173: 545-557, 2006.

25. Lin MY and Sheng ZH: Regulation of mitochondrial transport in neurons. Exp Cell Res 334: 35-44, 2015. 